\title{
To the Editor of the Mathematical Gazette
}

DEAR SIR,

To be provocative, may I suggest that one prophylactic against the disease which Mr. Hope-Jones depicts so vividly (XLII, 33) is the intelligent use of the very examples that $\mathrm{Mr}$ Wheeler deplores (XLII, 197)? If you insist too loudly that the circular functions of analysis have nothing to do with angles, you may find it hard to explain how the tables you rely on when you have a triangle to solve can be of the slightest use to you when you have an integral to evaluate.

Whatever the angular unit $\alpha$, an angle $\theta$ can be measured in terms of $\alpha$, that is, expressed as $t \alpha$ where $t$ is a real number, and if $y=\sin \theta$, then $y$ is a function of $t$ although the angle $\theta$ has been used to define the functional relation, just as the measure $h$ of my height in inches is a function of the measure $s$ of my age in years although without me there is no definition. There are two differences. As it happens, $y$ is already a pure number, and the problem of measurement does not come in on that side. Also you can not hope to get rid of me, if you are to relate $h$ to $s$ at all.

Of course we can not differentiate $f(\theta)$ with respect to $\theta$ or-which is the same thing $-f\left(x^{\circ}\right)$ with respect to $x^{\circ}$; we can not differentiate $h$ inches, or even $h$, with respect to $s$ years, but we can investigate $d h / d s$, and it is no less reasonable to investigate $d y / d t$. We find that $d y / d t=k_{\alpha} \cos \theta$, where $k_{\alpha}$ is independent of $t$, but depends on the choice of $\alpha$. If now we attempt to simplify an integral

$$
I=\int_{y_{1}}^{y_{2}} f(y) d y
$$

by the substitution $y=\sin \theta=\sin t \alpha$, we have

$$
I=k_{\alpha} \int_{t_{1}}^{t_{2}} f(\sin \theta) \cos \theta d t
$$

where $\sin t_{1} \alpha=y_{1}, \sin t_{2} \alpha=y_{2}$. A sine table does not, can not, show $\theta_{1}$ and $\theta_{2}$ as angles: it gives the measures of angles in terms of the unit adopted by the compiler We can use any table we can get hold of, but we must not forget $k_{\alpha}$, the differentiation factor associated with the unit.

The radian is the unit $\rho$ such that the differentiation factor $k_{\rho}$ is 1 . It is a highly artificial unit which no one but a mathematician would dream of introducing, and even the mathematician really does examples on garden rollers by counting revolutions and measuring the circumference. If $\theta=x \rho$, then $k_{\alpha} t=x$, whatever the unit $\alpha$. The inequality $\sin \theta<\tan \theta$ holds for an acute angle $\theta$; we can not insert $\theta$ between the extremes, but the uses to which

$$
\sin \theta<\theta<\tan \theta
$$

is commonly put are all served by $\sin \theta<x<\tan \theta$, without 
any measurement of arcs. With $\alpha$ for unit, $\rho$ is the angle whose measure is $1 / k_{\alpha}$, to define $\pi$ as the circular measure of a straight angle is equivalent to saying that with the straight angle for unit the differentiation factor is $\pi$. Reading in degrees we have to use the factor $\pi / 180$, and there is no difference between reading an angle in degrees as $18 \frac{7}{12}$ with differentiation factor $\pi / 180$ and reading the angle in minutes as 1115 with differentiation factor $\pi / 180 \times 60$.

Even if the radian is not given premature standing as a 'natural' unit, the question arises why horrible discoveries are left to the examiner to make. True, "Let us see if you can learn as well as we can teach" was the classical invitation of examiners, not of teachers. The teacher's pathetic belief that this year at least nobody in the class can have missed a point he has made so clear, survives shock after shock, and if perhaps he does forget sometimes that part of his job is to find out whether his confidence is justified, it is not fair to say that if his pupils give themselves away by accident to an examiner he has only himself to blame. It is not that he does not know that the whole argument has depended on the use of circular measure, or that he has not said so often enough. It is merely that for the purpose of verifying that there has been no misunderstanding, the usual examples, of indefinite integrals, of integrals with literal coefficients, of integrals devised to come out conveniently to recognisable fractions of $\pi$, and of numerical evaluations left in some such form as $3 \sin ^{-1} \frac{2}{3}+3-\sqrt{ } 5$, are utterly useless; full and accurate answers to these questions are compatible with a complete misapprehension unsuspected by teacher or taught, who are victims together of an almost unbroken tradition. The teacher who asks for the value to three significant figures of

$$
\int_{2}^{4} \sqrt{\left(\frac{7-x}{x-1}\right)} d x
$$

will learn the worst at once, but the usefulness of a question like this not as a test of arithmetical accuracy but as a probe is ignored even in M. A. Reports.

Probably Mr. Hope-Jones' first culprit thinks rough checks beneath the dignity of a student at the Advanced Level, but the reminder that the integral above is between $\frac{2}{1} \sqrt{ } \frac{5}{1}$ and $\frac{2}{1} \sqrt{ } \frac{3}{3}$, between $\frac{2}{2}\left(\sqrt{ } \frac{10}{2}+\sqrt{ } \frac{8}{4}\right)$ and $\frac{2}{2}\left(\sqrt{ } \frac{8}{4}+\sqrt{ } \frac{6}{6}\right)$, between $\frac{2}{3}\left(\sqrt{ } \frac{15}{3}+\sqrt{ } \frac{13}{5}+\sqrt{ } \frac{11}{7}\right)$ and $\frac{2}{3}\left(\sqrt{ } \frac{13}{5}+\sqrt{ } \frac{11}{7}+\sqrt{ } \frac{9}{9}\right)$, and so on, recalls attention to the very stuff of which the integral calculus is made, and the insult may slip through undetected.

Yours etc., E. H. Neville

P.S. Given two angles $\alpha, \theta$, the class of fractions $p / q$ such that $p \alpha$ is smaller than $q \theta$ is, in Russell's sense, a real number; this number is the measure of $\theta$ in terms of $\alpha$. 